\title{
Using Patient-Reported Outcome Measurement Information System Computer Adaptive Testing Domains to Investigate the Impact of Obesity on Physical Function, Pain Interference, and Mental Health in Sports Medicine Patients
}

\author{
Jacob W Blanchett, Noah A Kuhlmann, Mohsin S Fidai, Peter A Borowsky, Stephanie J Muh, Eric C Makhni* \\ Department of Orthopedic Surgery, Henry Ford Hospital, West Bloomfield, MI, USA
}

Background: While obesity has become an increasingly prevalent health concern in the United States, little emphasis has been placed on utilizing patient reported outcome measures (PROM) to investigate its impact on life from the patients' perspective. The purpose of the study was to determine the association between patients' body mass index (BMI) and three Patient-Reported Outcome Measurement Information System (PROMIS) computer adaptive test scores: upper extremity physical function (UE) or lower extremity physical function (PF), pain interference (PI), and depression (D).

Methods: Patients were recruited from two sports medicine orthopedic surgery clinics. PROMIS questionnaires were administered to patients arriving for their first visit. Patients were stratified into BMI groupings according to the National Institute of Health standards. Patients'BMl, sex, race, ethnicity, and injury were determined retroactively. Data were analyzed using a Pearson correlation and a least significant difference post hoc test.

Results: A total of 833 patients completed the set of PROMIS questionnaires that were retrospectively analyzed. $B M I$ was found to have a correlation with PROMIS-UE $(R=-0.111, P<0.05)$, PROMIS-PF $(R=-0.174, P<0.01)$, PROMIS-PI $(R=0.224, P<0.01)$, and PROMIS-D $(R=0.092, P<0.05)$. Obese patients also portrayed the worst PROMIS-UE, PROMIS-PI, and PROMIS-PF.

Conclusion: We found BMI to correlate with each PROMIS domain: negatively with PROMIS-UE, PROMIS-PF, PROMIS-D, and positively with PROMIS-PI. Additionally, overweight and obese BMI patients portrayed worse physical function and pain interference scores than their healthy group counterparts.

Key words: Body mass index, Patient reported outcome measures, Overweight, Obesity, Quality of life, Pain

\author{
Received May 31, 2019 \\ Reviewed August 9, 2019 \\ Accepted October 7, 2019 \\ ${ }^{*}$ Corresponding author \\ Eric C Makhni \\ (i) \\ https://orcid.org/0000-0003-4408-1175 \\ Department of Orthopedic Surgery, \\ Henry Ford Hospital, 6777 W Maple Rd, \\ West Bloomfield, MI 48322, USA \\ Tel: +1-248-661-6470 \\ Fax: +1-248-661-7155 \\ E-mail: emakhni1@hfhs.org
}

\section{INTRODUCTION}

Obesity, defined as a body mass index (BMI) of $30 \mathrm{~kg} / \mathrm{m}^{2}$ or higher, is a pressing health care problem around the world. It has been significantly associated with chronic disease in both men and women. ${ }^{1,2}$ Despite negative implications, obesity has become in- creasingly prevalent rising from $29.7 \%$ in 2009 to $32.4 \%$ in $2015 .{ }^{2}$ Studies have reported that statistics may actually underestimate the impact of obesity on mortality and its associated costs. ${ }^{1,3}$ Studies have addressed the impact of BMI on postoperative success in orthopedic surgery by utilizing objective measures, in addition to legacy patient reported outcome measures (PROM). ${ }^{4,5}$ However, the

Copyright (C) 2019 Korean Society for the Study of Obesity

() This is an Open Access article distributed under the terms of the Creative Commons Attribution Non-Commercial License (http://creativecommons.org/licenses/by-nc/4.0/) which permits unrestricted non-commercial use, distribution, and reproduction in any medium, provided the original work is properly cited. 
impact of BMI on physical function, pain interference, and depression in patients undergoing surgical or nonsurgical treatments has not been assessed from the patients' perspective utilizing the new Patient-Reported Outcome Measurement Information System's (PROMIS’s) computer adaptive testing (CAT) domains.

As healthcare continues to evolve to a more value-based approach, PROM are becoming increasingly important, as they represent clinical outcomes from the patient perspective. Many insurance companies have already begun replacing fee-for-service models with recently-developed, pay-for-performance models. ${ }^{6}$ These PROM also provide benefits to the patient, as patients are able to quantify progression throughout their treatment. ${ }^{7}$ The National Institute of Health (NIH) created PROMIS in a concentrated effort to streamline PROM reporting via a single effective and efficient system., PROMIS CAT forms are an electronic questionnaire that utilizes an item response theory to determine the most relevant subsequent question to ask — choosing from a large question bank specific to each subdomain. This has made PROMIS more responsive, had fewer ceiling and floor effects, and taken less time compared to legacy PROM. ${ }^{10,11}$ Additionally, several studies have found CAT forms to be well received by patients from a diverse population-even denoting that it can promote elevated self-reflection. ${ }^{12}$

The purpose of this study is to investigate the association of BMI on numerous health domains, such as physical function, pain interference (the impact of pain on a patient's quality of life), and mental health (depression) as measured by PROMIS CAT domains to enable providers to further incorporate $\mathrm{BMI}$ into treatment choices. We hypothesize that BMI has a negative correlation with physical function scores and a positive correlation with pain interference and depression scores.

\section{METHODS}

This study was performed under Institutional Board Approval of Henry Ford Health System (No. 11361), which waived the requirement for informed consent. All patients presenting to one of two surgeons' ambulatory sports medicine clinics, as part of routine practice, were asked to complete an electronic questionnaire. The questionnaire consisted of an intake form that presented injury location options, followed by the corresponding PROMIS CAT forms: PROMIS-upper extremity physical function (PROMISUE) or PROMIS-lower extremity physical function (PROMISPF), PROMIS-pain interference (PROMIS-PI), and PROMIS-depression (PROMIS-D). If a patient presented for an upper extremity complaint, they were directed to PROMIS-UE, whereas if a lower extremity complaint was selected, they were directed to PROMIS-PF. All patients were then automatically directed to PROMIS-PI and PROMIS-D forms. These questionnaires were administered on iPad tablets (Apple, Cupertino, CA, USA) using a secure, web-based application designed to support data capture for research studies hosted at our institution (REDCap, Nashville, TN, USA). ${ }^{13}$ The PROMIS CAT questionnaires were automatically scored on a standard system by REDCap; a score of 50 represents the average value of the general population with 10 points equal to one standard deviation change in that measure. ${ }^{14,15}$

Patients that presented for their first visit between June 30, 2017 and December 29, 2017, and completed the questionnaire, were retrospectively screened for inclusion. We screened a total of 858 patients and excluded a total of 25 patients for one of two reasons; 17 because they indicated both an upper extremity and lower extremity injury, and eight because they did not have a BMI indicated in their electronic medical record (EMR). In total, we included 833 of the 858 screened patients in our study. Several patient demographics were retrospectively collected from the EMR: BMI, age, sex, race, employment status, history of smoking and tobacco use, and an estimated median household income (MHI). Patients' BMI values were categorized as underweight, normal, overweight, and obese, according to accepted values provided by the NIH (Table 1 ). ${ }^{16}$ Tobacco use was documented as current, former, never, or unknown. Employment status was documented as either employed or unknown, with the latter encompassing both unemployed and unknown in the EMR. To determine the estimated MHI, we utilized patients' zip codes and the United States Census Bureau 2010 data

Table 1. BMl categorization $(\mathrm{n}=833)$

\begin{tabular}{llccc}
\hline BMl $\left(\mathrm{kg} / \mathrm{m}^{2}\right)$ & Group & PF value $(\mathrm{n})$ & UE value $(\mathrm{n})$ & Total $(\%)$ \\
\hline$<18.5$ & Underweight & 6 & 2 & 1 \\
$18.5-24.9$ & Healthy & 140 & 82 & 27 \\
$25.0-29.9$ & Overweight & 157 & 121 & 33 \\
$\geq 30.0$ & Obese & 189 & 136 & 39 \\
\hline
\end{tabular}

$\mathrm{BMI}$, body mass index; $\mathrm{PF}$, physical function; $\mathrm{UE}$, upper extremity. 
through an online platform, American Fact Finder. ${ }^{17}$

\section{Statistical analysis}

Summary statistics were calculated for the demographic data. Pearson correlations were calculated between BMI, and PROMISUE, PROMIS-PF, PROMIS-PI, and PROMIS-D. Additionally, each NIH-dictated BMI category was compared to one another through their respective PROMIS domain mean scores utilizing a least significant difference post hoc test. For each test, a $P$-value of $<0.05$ denoted statistical significance. All statistical analyses were conducted by a trained psychometrician.

\section{RESULTS}

A total of 833 unique patients completed the PROMIS CAT forms and met inclusion criteria for the study. The demographics of the patient cohort are described in Table 2. The average age of patients surveyed was 46.6 years (range, 13-89 years; standard deviation [SD], 18.5 years) with a male predominance (54\%). The average BMI was $29.4 \mathrm{~kg} / \mathrm{m}^{2}$ with a range of $17.7-58.9 \mathrm{~kg} / \mathrm{m}^{2}(\mathrm{SD}$,

Table 2. Patient demographic characteristics $(n=833)$

\begin{tabular}{|c|c|}
\hline Variable & Value \\
\hline Age (yr) & $46.6 \pm 18.5(13-89)$ \\
\hline $\mathrm{BMI}$ & $29.4 \pm 7.0(17.7-58.9)$ \\
\hline MHI (USD) & $62,576 \pm 24,222(21,415-157,536)$ \\
\hline \multicolumn{2}{|l|}{ Sex } \\
\hline Male & $448(54)$ \\
\hline Female & $385(46)$ \\
\hline \multicolumn{2}{|l|}{ Race } \\
\hline White & $519(62)$ \\
\hline Black & $172(21)$ \\
\hline Asian & $21(3)$ \\
\hline Other & $43(5)$ \\
\hline Unknown & $78(9)$ \\
\hline \multicolumn{2}{|c|}{ Employment status } \\
\hline Employed & $346(42)$ \\
\hline Unknown & $487(58)$ \\
\hline \multicolumn{2}{|l|}{ Tobacco use } \\
\hline Current & $96(12)$ \\
\hline Former & $185(22)$ \\
\hline Never & $530(64)$ \\
\hline Unknown & $22(3)$ \\
\hline
\end{tabular}

Values are presented as mean \pm standard deviation (range) or number (\%). BMI, body mass index; MHI, median household income; USD, United States dollar. $\left.7.0 \mathrm{~kg} / \mathrm{m}^{2}\right)$. Seventy-two percent of patients, according to their indicated BMI, were classified as either overweight or obese (Table 1). Sixty-two percent $(n=519)$ of patients identified as White and twenty-one percent $(n=172)$ as Black. Forty-two percent $(n=346)$ of patients had documented employment in the EMR, and the preponderance of patients had no history of tobacco use (64\%). There were 341 patients that presented with an upper extremity injury (the upper-extremity cohort) and 492 patients that presented with a lower extremity injury (the lower-extremity cohort) (Table 3). The most common upper and lower extremity diagnoses were rotator cuff tear and osteoarthritis, respectively.

We found BMI to correlate with each PROMIS domain: PROMIS-UE, PROMIS-PF, PROMIS-PI, and PROMIS-D. These established correlations indicate that for each point of increased BMI, the corresponding domain will change by the correlation coefficient. In upper extremity patients, BMI was found to have a correlation with PROMIS-UE $(\mathrm{R}=-0.111, P<0.05)$. In lower extremity patients, BMI was found to have a correlation with PROMIS-PF $(\mathrm{R}=$ $-0.174, P<0.01)$, PROMIS-PI $(\mathrm{R}=0.224, P<0.01)$, and PROMIS-D $(\mathrm{R}=0.092, P<0.05)$. There were no statistically significant

Table 3. Diagnoses $(n=833)$

\begin{tabular}{lc}
\hline Variable & No. (\%) \\
\hline Upper extremity cohort $(\mathrm{n}=341)$ & \\
Shoulder & \\
Rotator cuff & $121(15)$ \\
Instability/labrum & $26(3)$ \\
Nerve impingement & $22(3)$ \\
Osteoarthriti & $21(3)$ \\
Other & $99(12)$ \\
Elbow & \\
Lateral epicondylitis & $37(4)$ \\
Distal biceps & $8(1)$ \\
Other & $7(1)$ \\
Lower extremity cohort $(\mathrm{n}=492)$ & \\
Knee & \\
Osteoarthritis & $111(13)$ \\
Anterior cruciate ligament & $66(8)$ \\
Meniscus & $49(6)$ \\
Osteoarthritis & $34(4)$ \\
Other & $120(14)$ \\
Hip & \\
Femoroacetabular impingement & $37(4)$ \\
Osteoarthritis & $29(3)$ \\
Other & $46(6)$ \\
\hline
\end{tabular}


Table 4. Correlations between BMI and PROMIS domains

\begin{tabular}{lcccc}
\hline Variable & PROMIS-UE & PROMIS-PF & PROMIS-PI & PROMIS-D \\
\hline Upper extremity cohort BMI & $-0.111^{*}$ & & 0.063 & 0.012 \\
Lower extremity cohort BMI & & $-0.174^{\dagger}$ & $0.224^{\dagger}$ & $0.092^{*}$ \\
\hline
\end{tabular}

Pearson correlation (R-value).

Denotes a statistically significant finding, ${ }^{*} P<0.05 ;{ }^{\dagger} P<0.01$.

$B M I$, body mass index; PROMIS, Patient-Reported Outcomes Measurement Information System; UE, upper extremity physical function; PF, lower extremity physical function; Pl, pain interference; D, depression.

correlations found between BMI and PROMIS-PI or PROMIS-D in patients with an upper extremity injury (Table 4).

Both upper-extremity and lower-extremity patient cohorts exhibited statistically significant differences between BMI categorizations. For patients with upper extremity diagnoses, significant differences were found between overweight patients and obese patients when evaluating both PROMIS-UE and PROMIS-PI (33.7 vs. $31.1,60.8$ vs. $62.4 ; P<0.05)$. No statistical significance was found between any BMI categories with regard to PROMIS-D in upper or lower extremity patients. In lower extremity patients, there were statistically significant differences between healthy patients and both overweight and obese patients when assessing PROMIS-PF (41.7 vs. $39.9,41.7$ vs. $38.6 ; P<0.05)$. Lower extremity healthy patients also exhibited lower PI scores compared to obese patients (59.9 vs. 63.2; $P<0.05)$. Additionally, with regard to PI scores, overweight patients exhibited lower scores compared to their obese counterparts (61.3 vs. 63.2; $P<0.05)$ (Table 5).

\section{DISCUSSION}

This study, conducted in the ambulatory shoulder and sports medicine clinic, set out to determine the association between BMI and several PROMIS CAT domains: PROMIS-UE, PROMIS-PF, PROMIS-PI, and PROMIS-D. In upper extremity patients, we found a negative correlation between BMI and physical function and significantly better scores in the overweight group when compared to the obese group in regard to physical function and pain interference. In lower extremity patients, we found correlations between BMI and physical function, pain interference, and depression. Additionally, we found the healthy group to have significantly better physical function and pain interference scores when compared to the obese group.
Table 5. Impact of BMI categorization on PROMIS domain means

\begin{tabular}{lccll}
\hline Variable & PROMIS-UE & PROMIS-PF & PROMIS-PI & PROMIS-D \\
\hline Upper extremity cohort & & & & \\
$\quad$ Underweight & $27.5 \pm 8.3$ & & $68.4 \pm 2.1$ & $54.8 \pm 5.7$ \\
Normal & $33.2 \pm 8.5$ & $61.4 \pm 6.6$ & $49.5 \pm 9.0$ \\
Overweight & $33.7 \pm 8.9^{*}$ & $60.8 \pm 6.0^{*}$ & $48.1 \pm 9.4$ \\
Obese & $31.1 \pm 7.8^{*}$ & & $62.4 \pm 6.9^{*}$ & $49.1 \pm 10.1$ \\
Lower extremity cohort & & & & \\
Underweight & & $37.2 \pm 12.0$ & $63.7 \pm 7.5$ & $51.5 \pm 3.8$ \\
Normal & & $41.7 \pm 8.2^{*}$ & $59.9 \pm 7.5^{*}$ & $48.7 \pm 9.0$ \\
Overweight & & $39.9 \pm 7.4^{*}$ & $61.3 \pm 6.7^{*}$ & $49.0 \pm 10.4$ \\
Obese & $38.6 \pm 5.6^{*}$ & $63.2 \pm 6.1^{*}$ & $50.0 \pm 8.8$ \\
\hline
\end{tabular}

Values are presented as mean \pm standard deviation. Least significant difference post hoc test.

${ }^{*}$ Denotes a statistically significant finding $(P<0.05)$.

BMI, body mass index; PROMIS, Patient-Reported Outcomes Measurement Information System; UE, upper extremity physical function; PF, lower extremity physical function; $\mathrm{Pl}$, pain interference; $\mathrm{D}$, depression.

Elevated BMI was associated with a decrease in physical function for lower extremity patients. Our study found a statistically significant difference between healthy patients and both overweight and obese patients when assessing PROMIS-PF (41.7 vs. 39.9, 41.7 vs. 38.6; $P<0.05)$. These associations provide physicians with insight toward the impact of BMI on physical functioning. It is believed that an elevated BMI, particularly when reaching obese parameters, is accompanied with an increased amount of adipose tissue that compresses load-bearing joints. This compression results in inflammation and ultimately in the loss of muscle mass, and deterioration of the joint structures; collectively, over a period of time, these implications develop into a diminished level of physical functioning. ${ }^{18}$ Our study demonstrates similar findings to previously conducted studies. ${ }^{19,20}$ Although previous studies have attempted to quantify the association between BMI and physical function, there are contradictory findings in the literature. ${ }^{21-24}$ Many of the previous studies used legacy PROM which have been shown to have limitations. ${ }^{19,21,23,24}$ Our study utilized PROMIS CAT which has been shown to more precise than legacy PROM and have less floor and ceiling effects. ${ }^{10,25,26}$ Additionally, previous studies focused solely on postoperative physical function. The incorporation of all ambulatory patients in this study, regardless of treatment modality, allows for greater generalizability of the study findings.

Elevated BMI demonstrated an association with pain interference in our patient population. Specifically, in lower extremity patients, 
healthy patients exhibited lower pain interference scores compared to obese patients ( 59.9 vs. $63.2, P<0.05$ ) and overweight patients also exhibited lower scores compared to their obese counterparts (61.3 vs. 63.2, $P<0.05)$. These associations provide insight toward the impact of BMI on pain interference. It is believed that both increased mechanical stress and increased amounts of adipose tissue can lead to pain. The mechanical stress of overloading the knee, hip, and back, can cause both injury and degradation of these structures. In addition, both the increased size and number of adipocytes result in an enhanced inflammatory response; this is due to elevated levels of chemical mediators that interact with the nervous system to create the perception of pain: kinins, prostaglandins, and histamine..$^{27-29}$ The association our study found is comparable to that which has been previously documented in prior studies. ${ }^{25,30}$ However, these studies used legacy PROM measures and questionnaires during different periods of postoperative treatment. Our study, as mentioned, utilized PROMIS CAT forms and was administered to all new patients presenting to the ambulatory sports medicine clinic.

With the appeal of NIH PROMIS and its potential to standardize PROM, the documentation of cohort scores and the factors that impact them are of vital importance. To date, in the ambulatory sports medicine clinic, there have not been any documented PROMIS domain scores reported for each respective BMI group. Our study provides valuable information to establish the association between BMI and the PROMIS CAT domains for upper and lower extremity patients. The scores established by our study for each BMI group give providers an enhanced ability to compare PROM scores beyond the generic reference population, to patients within the same BMI group. Moving forward with longitudinal studies of patients within their respective BMI groups, together with this information, could potentially enable physicians to better comprehend patients' PROMIS scores and better predict their expected improvement following specific treatment options-improving the viability of PROMIS and result in more effective and efficient personalized treatments. Additionally, further studies that address the responsiveness of the PROMIS CAT domains to longitudinal weight changes during specific treatment options could further benefit the medical community in determining the most effective individualized treatment.

\section{Limitations}

There are several limitations noted for our study. First, we did not control for some potentially confounding variables: age, sex, race, diagnosis. Secondly, there was a relatively small number of underweight patients included in this study, therefore making it challenging to statistically analyze the PROMIS CAT scores in this group. Existing literature has shown that underweight individuals exhibit similar statistics to overweight and obese individuals, each worse than those with a healthy BMI. ${ }^{31,32}$ Additionally, we did not use any objective measures in our outcome reporting to address physical function, pain interference, or depression. As many studies have validated PROMIS CAT measures against traditional, diagnosis-specific PROM, ${ }^{33-39}$ our practice is to measure PROMIS CAT domains only, as these measures are more efficient to administer than corresponding legacy scores. ${ }^{10}$ Lastly, we only utilized the English language version of the PROMIS CAT forms. Thus, the study may not be completely generalizable to non-English populations. Despite this caveat, a broad range of socioeconomic diversity was encompassed within this study, as is evident by the demographic MHI and race distributions.

In conclusion, this study established that patients presenting to the ambulatory sports medicine clinic demonstrate several significant adverse associations between BMI, and PROMIS-PF, PROMIS-UE, PROMIS-PI, and PROMIS-D. Each of these findings verify that PROMIS CAT forms can be used as an efficient yet effective method to increase quality of care and to further research the impact between BMI and many aspects of life.

\section{CONFLICTS OF INTEREST}

One or more of the authors has declared the following potential conflict of interest or source of funding: SJM is a paid consultant for DePuy and Exactech; has received educational support from Arthrex; and has received hospitality payments from DePuy Synthes, Zimmer Biomet Holdings, Biomet Orthopedics, Exactech, Tornier, Conventus Orthopaedics, and Arthrex. ECM receives royalties from Springer, has received educational support from Pinnacle and Smith \& Nephew, and has received hospitality payments from Smith \& Nephew and Stryker. Other authors declare no potential conflicts of interest. 


\section{ACKNOWLEDGMENTS}

This study was internally funded by the Department of Orthopaedic Surgery at the Henry Ford Health System.

\section{AUTHOR CONTRIBUTIONS}

Study concept and design: ECM, SJM, JWB; acquisition of data: JWB, PAB; analysis and interpretation of data: JWB, NAK, MSF; drafting of the manuscript: JWB, NAK, PAB; critical revision of the manuscript: ECM, MSF, SJM; and study supervision: ECM, SJM.

\section{REFERENCES}

1. Masters RK, Reither EN, Powers DA, Yang YC, Burger AE, Link BG. The impact of obesity on US mortality levels: the importance of age and cohort factors in population estimates. Am J Public Health 2013;103:1895-901.

2. Seo MH, Kim YH, Han K, Jung JH, Park YG, Lee SS, et al. Prevalence of obesity and incidence of obesity-related comorbidities in Koreans based on National Health Insurance Service health checkup data 2006-2015. J Obes Metab Syndr 2018;27: 46-52.

3. Krueger PM, Coleman-Minahan K, Rooks RN. Race/ethnicity, nativity and trends in BMI among U.S. adults. Obesity (Silver Spring) 2014;22:1739-46.

4. Murray DW, Pandit H, Weston-Simons JS, Jenkins C, Gill HS, Lombardi AV, et al. Does body mass index affect the outcome of unicompartmental knee replacement? Knee 2013;20:461-5.

5. Jameson SS, Mason JM, Baker PN, Elson DW, Deehan DJ, Reed MR. The impact of body mass index on patient reported outcome measures (PROMs) and complications following primary hip arthroplasty. J Arthroplasty 2014;29:1889-98.

6. Squitieri L, Bozic KJ, Pusic AL. The role of patient-reported outcome measures in value-based payment reform. Value Health 2017;20:834-6.

7. Deshpande PR, Rajan S, Sudeepthi BL, Abdul Nazir CP. Patient-reported outcomes: a new era in clinical research. Perspect Clin Res 2011;2:137-44.
8. Makhni EC. Editorial commentary: are we comparing apples to oranges? The need for standardized outcomes reporting in orthopaedic surgery. Arthroscopy 2017;33:484-5.

9. Baumhauer JF. Patient-reported outcomes: are they living up to their potential? N Engl J Med 2017;377:6-9.

10. Scott EJ, Westermann R, Glass NA, Hettrich C, Wolf BR, Bollier MJ. Performance of the PROMIS in patients after anterior cruciate ligament reconstruction. Orthop J Sports Med 2018; 6:2325967118774509.

11.Hung M, Stuart AR, Higgins TF, Saltzman CL, Kubiak EN. Computerized adaptive testing using the PROMIS physical function item bank reduces test burden with less ceiling effects compared with the short musculoskeletal function assessment in orthopaedic trauma patients. J Orthop Trauma 2014;28: 439-43.

12. Kasturi S, Burket JC, Berman JR, Kirou KA, Levine AB, Sammaritano LR, et al. Feasibility of Patient-Reported Outcomes Measurement Information System (PROMIS ${ }^{\circledR}$ ) computerized adaptive tests in systemic lupus erythematosus outpatients. Lupus 2018;27:1591-9.

13. Harris PA, Taylor R, Thielke R, Payne J, Gonzalez N, Conde JG. Research electronic data capture (REDCap): a metadatadriven methodology and workflow process for providing translational research informatics support. J Biomed Inform 2009; 42:377-81.

14. Cella D, Gershon R, Lai JS, Choi S. The future of outcomes measurement: item banking, tailored short-forms, and computerized adaptive assessment. Qual Life Res 2007;16 Suppl 1:133-41.

15. Cella D, Yount S, Rothrock N, Gershon R, Cook K, Reeve B, et al. The Patient-Reported Outcomes Measurement Information System (PROMIS): progress of an NIH Roadmap cooperative group during its first two years. Med Care 2007;45(5 Suppl 1):S3-11.

16. Centers for Disease Control and Prevention. About adult BMI [Internet]. Atlanta, GA: Centers for Disease Control and Prevention; 2017 [cited 2019 Dec 2]. Available from: https://www. cdc.gov/healthyweight/assessing/bmi/adult_bmi/

17. American Fact Finder. United States Census Bureau 2010 [Internet]. Washington, DC: United States Census Bureau; 2010 
[cited 2019 Dec 2]. Available from: https://factfinder.census. gov/faces/nav/jsf/pages/community_facts.xhtml?src=bkmk

18. Vincent HK, Heywood K, Connelly J, Hurley RW. Obesity and weight loss in the treatment and prevention of osteoarthritis. PM R 2012;4(5 Suppl):S59-67.

19. Kowalchuk DA, Harner CD, Fu FH, Irrgang JJ. Prediction of patient-reported outcome after single-bundle anterior cruciate ligament reconstruction. Arthroscopy 2009;25:457-63.

20. Saltzman BM, Kuhns BD, Basques B, Leroux T, Alter J, Mather RC 3rd, et al. The influence of body mass index on outcomes after hip arthroscopic surgery with capsular plication for the treatment of femoroacetabular impingement. Am J Sports Med 2017;45:2303-11.

21. Foran JR, Mont MA, Etienne G, Jones LC, Hungerford DS. The outcome of total knee arthroplasty in obese patients. J Bone Joint Surg Am 2004;86:1609-15.

22. O’Neill SC, Butler JS, Daly A, Lui DF, Kenny P. Effect of body mass index on functional outcome in primary total knee arthroplasty: a single institution analysis of 2180 primary total knee replacements. World J Orthop 2016;7:664-9.

23. Fabricant PD, Rosenberger PH, Jokl P, Ickovics JR. Predictors of short-term recovery differ from those of long-term outcome after arthroscopic partial meniscectomy. Arthroscopy 2008;24: 769-78.

24. Rosenberger PH, Dhabhar FS, Epel E, Jokl P, Ickovics JR. Sex differences in factors influencing recovery from arthroscopic knee surgery. Clin Orthop Relat Res 2010;468:3399-405.

25. Giesinger JM, Loth FL, MacDonald DJ, Giesinger K, Patton JT, Simpson AH, et al. Patient-reported outcome metrics following total knee arthroplasty are influenced differently by patients' body mass index. Knee Surg Sports Traumatol Arthrosc 2018;26:3257-64.

26. Ballal MS, Khan Y, Hastie G, Hatcher A, Coogan S, McNicholas MJ. Functional outcome of primary hamstring anterior cruciate ligament reconstruction in patients with different body mass index classes. Arthroscopy 2013;29:1314-21.

27. Watkins LR, Maier SF, Goehler LE. Immune activation: the role of pro-inflammatory cytokines in inflammation, illness responses and pathological pain states. Pain 1995;63:289-302.

28. Dray A. Inflammatory mediators of pain. Br J Anaesth 1995;
75:125-31.

29. Liewluck T, Saperstein DS. Progressive muscular atrophy. Neurol Clin 2015;33:761-73.

30. Heuch I, Hagen K, Heuch I, Nygaard Ø, Zwart JA. The impact of body mass index on the prevalence of low back pain: the HUNT study. Spine (Phila Pa 1976) 2010;35:764-8.

31. Harada K, Doki N, Hagino T, Miyawaki S, Ohtake S, Kiyoi H, et al. Underweight status at diagnosis is associated with poorer outcomes in adult patients with acute myeloid leukemia: a retrospective study of JALSG AML 201. Ann Hematol 2018;97: 73-81.

32. Kress AM, Peterson MR, Hartzell MC. Association between obesity and depressive symptoms among U.S. Military active duty service personnel, 2002. J Psychosom Res 2006;60:26371.

33. Anthony CA, Glass N, Hancock K, Bollier M, Hettrich CM, Wolf BR. Preoperative performance of the patient-reported outcomes measurement information system in patients with rotator cuff pathology. Arthroscopy 2017;33:1770-4.

34. Anthony CA, Glass NA, Hancock K, Bollier M, Wolf BR, Hettrich CM. Performance of PROMIS instruments in patients with shoulder instability. Am J Sports Med 2017;45:449-53.

35. Beckmann JT, Hung M, Bounsanga J, Wylie JD, Granger EK, Tashjian RZ. Psychometric evaluation of the PROMIS Physical Function Computerized Adaptive Test in comparison to the American Shoulder and Elbow Surgeons score and Simple Shoulder Test in patients with rotator cuff disease. J Shoulder Elbow Surg 2015;24:1961-7.

36. Dowdle SB, Glass N, Anthony CA, Hettrich CM. Use of PROMIS for patients undergoing primary total shoulder arthroplasty. Orthop J Sports Med 2017;5:2325967117726044.

37. Kazmers NH, Hung M, Rane AA, Bounsanga J, Weng C, Tyser AR. Association of physical function, anxiety, and pain interference in nonshoulder upper extremity patients using the PROMIS platform. J Hand Surg Am 2017;42:781-7.

38. Minoughan CE, Schumaier AP, Fritch JL, Grawe BM. Correlation of PROMIS Physical Function Upper Extremity Computer Adaptive Test with American Shoulder and Elbow Surgeons shoulder assessment form and Simple Shoulder Test in patients with shoulder arthritis. J Shoulder Elbow Surg 2018; 


\section{7:585-91.}

39. Minoughan CE, Schumaier AP, Fritch JL, Grawe BM. Correlation of patient-reported outcome measurement information system physical function upper extremity computer adaptive testing, with the American Shoulder and Elbow Surgeons shoulder assessment form and Simple Shoulder Test in patients with shoulder pain. Arthroscopy 2018;34:1430-6. 